OPEN ACCESS

Edited by:

Salvatore Andrea Mastrolia, Ospedale dei Bambini Vittore Buzzi,

Italy

Reviewed by:

Asnat Walfisch

Hadassah Medical Center, Israel Ana Isabel Padilla-Pérez,

Universidad de La Laguna, Spain

*Correspondence:

Reut Rotem

reutah86@gmail.com

Specialty section: This article was submitted to Neonatology,

a section of the journal

Frontiers in Pediatrics

Received: 07 January 2019 Accepted: 25 February 2019

Published: 19 March 2019

Citation:

Blum M, Weintraub AY, Baumfeld Y, Rotem $R$ and Pariente G (2019)

Perinatal Outcomes of Small for

Gestational Age Neonates Born With an Isolated Single Umbilical Artery.

Front. Pediatr. 7:79.

doi: 10.3389/fped.2019.00079

\section{Perinatal Outcomes of Small for Gestational Age Neonates Born With an Isolated Single Umbilical Artery}

\author{
Maayan Blum ${ }^{1}$, Adi Y. Weintraub ${ }^{1}$, Yael Baumfeld ${ }^{1}$, Reut Rotem ${ }^{2 *}$ and Gali Pariente ${ }^{1}$ \\ ${ }^{1}$ Department of Obstetrics and Gynecology, Soroka University Medical Center, Ben-Gurion University of the Negev, \\ Beer-Sheva, Israel, ${ }^{2}$ Department of Obstetrics and Gynecology, Shaare Zedek Medical Center, Affiliated with the Hebrew \\ University Medical School of Jerusalem, Jerusalem, Israel
}

Objective: To investigate pregnancy outcomes of small for gestational age (SGA) neonates born with isolated single umbilical artery (iSUA) compared to SGA neonates without iSUA.

Study Design: This was a population-based retrospective cohort analysis. The study group was defined as a singleton SGA neonate born with iSUA, while an SGA neonate without iSUA comprised the comparison group. We evaluated adverse perinatal outcomes in all SGA neonates born at the Soroka University Medical Center between the years 1998-2013. Multiple gestations, fetuses with known congenital malformations or chromosomal abnormalities and patients with lack of prenatal care were excluded from the study. Multivariate logistic regression models were constructed to identify independent factors associated with adverse perinatal outcomes.

Results: Of 12,915 SGA deliveries, 1.2\% (162) were complicated with iSUA. Women in the study group were older with a significantly lower gestational age at delivery compared with the comparison group. Rates of women who conceived after infertility treatments were higher in the study group. Additionally, patients in the study group had significantly higher rates of preterm deliveries, placental abruption, cord prolapse, non-reassuring fetal heart rates and cesarean delivery were noted in the study group. These neonates had a significantly lower birth weight (1988.0 \pm 697 vs. $2388.3 \pm 481 p<0.001)$ and higher rates of low APGAR scores at the first and fifth minutes after birth compared with controls. Perinatal mortality was also found to be significantly higher among SGA neonates complicated with iSUA. Preterm delivery as well as perinatal mortality were found independently associated with iSUA among SGA neonates (aOR 4.01, 95\% Cl 2.88-5.59, aOR 2.24, 95\% Cl 1.25-4.01, respectively).

Conclusion: SGA pregnancies complicated with iSUA are at higher risk for adverse pregnancy and perinatal outcomes as compared to SGA pregnancies without iSUA.

Keywords: small for gestational age, isolated umbilical artery, perinatal mortality, preterm delivery, outcomes 


\section{INTRODUCTION}

The umbilical cord is formed between the 13 and 38th day following conception and contains two umbilical arteries and one umbilical vein (1). Single umbilical artery (SUA) is defined as the absence of one umbilical artery and is considered the most common macroscopic anomaly of the placenta and the most common malformation of the umbilical cord (2). SUA can occur due to aplasia or as a consequence of atrophy of one of the arteries (3). The incidence of SUA is $0.2-1.6 \%$ in euploid fetuses compared with $9-11 \%$ in aneuploid fetuses. The reported prevalence of SUA is $4.6 \%$ of twin births and $1 \%$ of singletons $(3,4)$. SUA is defined isolated SUA (iSUA), if no additional chromosomal or structural abnormalities occur (5). Fetuses with an iSUA are at increased risk for intra-uterine growth restriction (IUGR) during pregnancy and small for gestational age (SGA) at birth $(4,6)$.

SUA has been associated with various malformations and pathologies such as cardiac malformations, cleft lip or palate, esophageal atresia, ano-rectal atresia/stenosis (7) and adverse pregnancy outcomes such as preterm birth, diabetes, epilepsy, preeclampsia, polyhydramnios, and oligohydramnios (3). The incidence of chromosomal defects in fetuses with SUA is considerably higher, specifically noting trisomy 18 with a 7 fold increased risk (8). SUA is rare in Mendelian disorders but relatively frequent in idiopathic anomalies (9). iSUA has been reported as an independent risk factor for perinatal mortality(10), cesarean section (11) and adverse pregnancy outcomes (12). Hence induction of labor at 40 weeks of gestation for iSUA fetuses has been recommended (10).

By definition, $10 \%$ of all live births are born SGA. Neonates are considered small for gestational age if they are smaller than the 10th percentile with regard to the anthropometric index being used (13). SGA neonates in comparison with appropriate for gestational age (AGA) neonates are considered to have an increased risk of complications such as bronchopulmonary dysplasia and adverse neurodevelopmental outcomes, as well as adverse pregnancy outcomes (14). Gutvirtz et al. presented iSUA as an independent risk factor for adverse perinatal outcomes in term neonates with normal estimated fetal weight prior to delivery (12). In our study, we focused on SGA neonates born with iSUA since little is known regarding this specific combination. The goal of this study was to examine the pregnancy outcomes of those SGA neonates born with iSUA compared to SGA neonates without iSUA.

\section{MATERIALS AND METHODS}

In this population-based retrospective cohort analysis, all deliveries of singleton SGA neonates occurring between the years 1998 and 2014 were included. The study was conducted at the Soroka University Medical Center (SUMC), which is the only tertiary medical center in southern Israel where virtually all births to women in southern Israel take place. Due to the diversity of this population, we believe that this cohort represents a non-selective population-based data. The institutional review board of SUMC approved the study in accordance with the
Helsinki declaration (\# SOR-0372-17). In complying with the Israeli Ministry of Health regulations, the institutional ethics committee did not require written informed consent because the data was obtained anonymously from medical records, with no direct participation or involvement of patients and public.

Case records of SGA neonates with iSUA were compared with SGA neonates without iSUA. The diagnosis of iSUA was confirmed following labor, as per protocol at SUMC where the midwives routinely examine the placenta and umbilical cord immediately after delivery. Thus, all cases of iSUA are confirmed by a physical examination.

In order to fit the definition of isolated SUA, we excluded from the cohort neonates that were AGA or large for gestational age neonates (LGA), multiple gestations, fetuses with known congenital malformations or chromosomal abnormalities and patients with lack of prenatal care. Those outcomes assessed included maternal characteristics, pregnancy characteristics, labor and delivery characteristics, and neonatal outcomes.

Postpartum hemorrhage is defined as the loss of $500 \mathrm{ml}$ of blood following a vaginal birth, $1 \mathrm{~L}$ of blood following a cesarean section or a drop in the maternal hemoglobin of $3 \mathrm{gr} \%$ after delivery. Perinatal mortality includes one of the following occurrences: Intrauterine fetal death which is defined as the death of a fetus older than 22 weeks gestation, intrapartum death which is defined as the death of a neonate during delivery or postpartum death which is the death of a neonate 1 month after birth.

Data was collected from the computerized perinatal database of the Obstetrics and Gynecology department of the SUMC.

\section{Statistical Analysis}

Statistical analysis was performed using SPSS 21.0 (SPSS, Chicago, IL). Initial analysis included descriptive statistics followed by advanced analytical statistics tests. Normal distributed continuous variables are presented as mean \pm standard deviation, comparison between the study groups was done using $t$-test. Continuous variables which are not normally distributed are presented as median with interquartile range, Mann Whitney test was used for their statistical analysis. Categorical variables are presented in counts and percentages, Chi-Square or Fisher Exact test when appropriate were used. All analysis with two-sided and $p$-value of 0.05 was considered significant. Multivariate logistic regression models were constructed in order to identify independent risk factors associated with the selected outcomes.

\section{RESULTS}

Of 12,915 SGA deliveries that were included in the analysis, $1.2 \%$ (162) were complicated with iSUA. Table 1 presents demographic and clinical characteristics of patients delivering SGA neonates with iSUA (study group) in comparison with patients delivering SGA neonates without iSUA (comparison group). Women in the study group were older with a significantly lower gestational age at delivery. Rates of women who conceived after infertility treatments and rates of women with habitual abortions were higher in the study group. 
TABLE 1 | Demographic and clinical characteristics of patients delivering an SGA infant with and without iSUA.

\begin{tabular}{|c|c|c|c|}
\hline Characteristics & $\begin{array}{l}\text { SGA with iSUA } \\
\quad(n=162)\end{array}$ & $\begin{array}{l}\text { SGA without iSUA } \\
\qquad(n=12,915)\end{array}$ & $P$-values \\
\hline $\begin{array}{l}\text { Maternal age years } \\
(\text { mean }+\mathrm{SD})\end{array}$ & $28.14 \pm 5.82$ & $27.43 \pm 5.95$ & 0.04 \\
\hline $\begin{array}{l}\text { Gravidity } n(\%) \\
1 \\
2-4 \\
5 \leq\end{array}$ & $\begin{array}{l}52(32.1) \\
65(40.1) \\
45(27.8)\end{array}$ & $\begin{array}{l}4,067(31.5) \\
5,562(43.1) \\
3,282(25.4)\end{array}$ & 0.71 \\
\hline Parity $n(\%)$ & & & 0.37 \\
\hline 1 & $64(39.8)$ & 4,765 (36.9) & \\
\hline $2-4$ & $62(38.5)$ & $5,675(44.0)$ & \\
\hline $5 \leq$ & $35(21.7)$ & 2,472 (19.1) & \\
\hline $\begin{array}{l}\text { Gestational age at delivery } \\
\text { (mean }+ \text { SD) }\end{array}$ & $36.80 \pm 3.80$ & $38.60 \pm 2.65$ & $<0.001$ \\
\hline $\begin{array}{l}\text { Previous cesarean delivery } \\
n(\%)\end{array}$ & $22(13.6)$ & $1,614(12.5)$ & 0.68 \\
\hline $\mathrm{BOH} n(\%)$ & $8(4.9)$ & $608(4.7)$ & 0.85 \\
\hline Habitual abortion $n(\%)$ & $15(9.3)$ & $684(5.3)$ & 0.03 \\
\hline $\begin{array}{l}\text { Pregnancy after infertility } \\
\text { treatment } n(\%)\end{array}$ & $15(9.3)$ & 334 (2.6) & $<0.001$ \\
\hline Gender $n(\%)$ & & & 0.44 \\
\hline Male & 64 (39.5) & $4,723(36.6)$ & \\
\hline Female & $98(60.5)$ & $8,192(63.4)$ & \\
\hline
\end{tabular}

SGA, small for gestational age; iSUA, isolated umbilical artery; $\mathrm{BOH}$, bad obstetric history.

TABLE 2 | Characteristics of pregnancy complications of SGA neonates with and without iSUA.

\begin{tabular}{|c|c|c|c|}
\hline Characteristics & $\begin{array}{c}\text { SGA with iSUA } \\
n=162\end{array}$ & $\begin{array}{c}\text { SGA without } \\
\text { iSUA } n=12,915\end{array}$ & $\boldsymbol{P}$-values \\
\hline Preterm deliveries $n(\%)$ & $61(37.7)$ & $1,579(12.2)$ & $<0.001$ \\
\hline $\begin{array}{l}\text { Pre-gestational and } \\
\text { gestational diabetes mellitus } \\
n(\%)\end{array}$ & $8(4.9)$ & 467 (3.6) & 0.37 \\
\hline $\begin{array}{l}\text { Amnion fluid abnormalities } \\
\text { Polyhydramnios } n(\%) \\
\text { Oligohydramnios } n(\%)\end{array}$ & $\begin{array}{l}17(10.5) \\
21(13.0)\end{array}$ & $\begin{array}{l}302(2.3) \\
1,087(8.4)\end{array}$ & $\begin{array}{c}<0.001 \\
0.04\end{array}$ \\
\hline Placenta Previa $n$ (\%) & $2(1.2)$ & $60(0.5)$ & 0.16 \\
\hline PROM n (\%) & $8(4.9)$ & $1,048(8.1)$ & 0.14 \\
\hline $\begin{array}{l}\text { Hypertensive disorders in } \\
\text { pregnancy } n(\%)\end{array}$ & $23(14.2)$ & $1,347(10.4)$ & 0.12 \\
\hline
\end{tabular}

SGA, small for gestational age; iSUA, isolated umbilical artery; PROM, premature rupture of membranes. Hypertensive disorders in pregnancy included chronic hypertension, preeclampsia and severe preeclampsia.

Table 2 compares gestational characteristics and pregnancy complications of pregnancies complicated with SGA neonates with and without iSUA. Pregnancies in the study group had significantly higher rates of preterm deliveries (37.7 vs. $12.2 \%$, $p<0.001$ ) and amniotic fluid abnormalities (10.5 vs. $2.3 \%$, $p<0.001$ and 13.0 vs. $8.4 \% p=0.04$ for polyhydramnios and oligohydramnios, respectively) as compared to pregnancies of the comparison group.

Labor and delivery characteristics and neonatal outcomes of the two groups are presented in Table 3. Pregnancies in the
TABLE 3 | Labor and delivery characteristics and neonatal outcomes of patients delivering an SGA infant with and without iSUA.

\begin{tabular}{|c|c|c|c|}
\hline Characteristics & $\begin{array}{c}\text { SGA with } \\
\text { iSUA } n=162\end{array}$ & $\begin{array}{c}\text { SGA without iSUA } \\
n=12,915\end{array}$ & $P$-values \\
\hline Placental abruption $n(\%)$ & $7(4.3)$ & $192(1.5)$ & 0.01 \\
\hline Cord prolapse $n(\%)$ & $7(4.3)$ & $61(0.5)$ & $<0.001$ \\
\hline $\begin{array}{l}\text { Meconium in amniotic fluid } \\
n(\%)\end{array}$ & 27 (16.5) & $2,383(18.5)$ & 0.56 \\
\hline Induction of labor $n(\%)$ & $26(16)$ & $1,469(11.4)$ & 0.06 \\
\hline $\begin{array}{l}\text { Mode of deliver } \\
\text { Cesarean section } n(\%) \\
\text { Vacuum } n(\%)\end{array}$ & $\begin{array}{c}59(36.4) \\
8(4.9)\end{array}$ & $\begin{array}{l}2,618(20.3) \\
452(3.5)\end{array}$ & $\begin{array}{c}<0.001 \\
0.32\end{array}$ \\
\hline $\begin{array}{l}\text { Non-reassuring fetal heart } \\
\text { rate } n(\%)\end{array}$ & $14(8.6)$ & $615(4.8)$ & 0.02 \\
\hline $\mathrm{PPH} n(\%)$ & $1(0.6)$ & $59(0.5)$ & 0.76 \\
\hline $\begin{array}{l}\text { Infant characteristics Birth } \\
\text { weight gr (Mean } \pm \text { SD) }\end{array}$ & $1988.00 \pm 697.88$ & $2388.33 \pm 481.22$ & $<0.001$ \\
\hline APGAR $1 \mathrm{~min}<7 n(\%)$ & 32 (19.6) & $1,389(10.8)$ & $<0.001$ \\
\hline APGAR $5 \mathrm{~min}<7 n(\%)$ & $10(6.2)$ & $297(2.3)$ & $<0.001$ \\
\hline $\mathrm{PH}<7 n(\%)$ & $0(0.0)$ & $6(0.05)$ & 1.00 \\
\hline Perinatal mortality $n(\%)$ & 20 (12.3) & $420(3.3)$ & $<0.001$ \\
\hline $\begin{array}{l}\text { Gender (\%) } \\
\text { Male } \\
\text { Female }\end{array}$ & $\begin{array}{l}64(39.5) \\
98(60.5)\end{array}$ & $\begin{array}{l}4,723(36.6) \\
8,192(63.4)\end{array}$ & 0.44 \\
\hline
\end{tabular}

SGA, small for gestational age; iSUA, isolated umbilical artery; SD, standard deviation; $\mathrm{PPH}$, postpartum hemorrhage.

study group had higher rates of placental abruption (4.3 vs. $1.5 \%, p=0.01)$, cord prolapse $(4.3$ vs. $0.5 \%, p<0.001)$, nonreassuring fetal heart rate patterns ( 8.6 vs. $4.8 \% p=0.02)$ and cesarean section $(36.4$ vs. $20.3 \% p<0.001)$. These neonates had significantly lower birth weight $(1988.0 \pm 697$ vs. $2388.3 \pm 481$ $p<0.001)$ and higher rates of low APGAR score at the first and fifth minutes after birth compared with the comparison group. Perinatal mortality was also significantly higher in SGA neonates complicated with iSUA (12.3 vs. $3.3 \%, p<0.001)$. Induction of labor was found to be higher in the study group (16\%) compared to the comparison group (11.4\%), though this was not found to be significant $(p=0.06)$.

In order to assess whether the increased risk for preterm delivery was independently associated with iSUA, a multivariate logistic regression model controlling for maternal age, fertility treatments and placental abruption was constructed, with preterm delivery as the outcome variable (Table 4). iSUA, was found to be independently associated with preterm delivery (adjusted OR 4.01, 95\% CI 2.88-5.59, $p<0.001$, Table 4).

Another multivariate logistic regression model was constructed, controlling for gestational age at birth, to estimate an independent association with perinatal mortality (Table 5). iSUA was found to be independently associated with perinatal mortality (adjusted OR 2.24, 95\% CI 1.25-4.01, $p=0.01$, Table 5).

\section{DISCUSSION}

In this large population based cohort study, there were 162 neonates born with iSUA, which is $0.01 \%$ of the entire population 
TABLE 4 | Multivariate logistic regression for the prediction of preterm delivery.

\begin{tabular}{llcl}
\hline Variable & OR & $\mathbf{9 5 \%} \mathbf{~ C l}$ & $\boldsymbol{P}$-value \\
\hline $\begin{array}{l}\text { iSUA in SGA neonate vs. no iSUA in SGA } \\
\text { neonate }\end{array}$ & 4.01 & $2.88-5.59$ & $<0.001$ \\
Maternal age & 1.03 & $1.02-1.03$ & $<0.001$ \\
$\begin{array}{l}\text { Fertility treatment } \\
\text { Placental abruption }\end{array}$ & 1.92 & $1.48-2.50$ & $<0.001$ \\
\hline $\begin{array}{l}\text { OR, odds ratio; Cl, confidence interval; SUA, single umbilical artery; SGA, small for } \\
\text { gestational age. }\end{array}$ & & &
\end{tabular}

TABLE 5 | Multivariate logistic regression for the prediction of perinatal mortality.

\begin{tabular}{lccc}
\hline Variable & OR & $\mathbf{9 5 \%} \mathbf{C l}$ & $\boldsymbol{P}$-value \\
\hline $\begin{array}{l}\text { iSUA in SGA neonate vs. no iSUA in SGA } \\
\text { neonate }\end{array}$ & 2.24 & $1.25-4.01$ & 0.01 \\
$\begin{array}{l}\text { Gestational age at delivery } \\
\text { nat }\end{array}$ & 0.69 & $0.67-0.70$ & $<0.001$
\end{tabular}

OR, odds ratio; $\mathrm{Cl}$, confidence interval; SUA, single umbilical artery.

of the study. This rate is lower than the incidence of SUA reported in the literature $[0.02-1.6 \%(4)]$, possibly due to the latter referring to the rate of non-isolated SUA. Delivery of an SGA neonate born with iSUA was found to be an independent risk factor for preterm delivery and perinatal mortality. As previously mentioned, our discoveries show a substantially higher rate of preterm delivery and perinatal mortality in SGA neonates complicated with iSUA.

Friebe-Hoffmann et al. demonstrated that $5.1 \%$ of neonates born with an isolated single umbilical artery had been delivered preterm (15). Others have reported a preterm rate of $11.8 \%$ (16). Both rates are significantly lower than in this study $(37.7 \%)$ who were born preterm and had both iSUA and SGA. Battarbee et al. showed an increased incidence of preterm birth among pregnancies with iSUA emphasizing the significant increase of preterm deliveries due to medical indications, which may be explained by their findings of increased incidence of gestational hypertension and preeclampsia amongst these pregnancies (17). This, along with the tendency of obstetricians to induce labor earlier in pregnancies with fetuses who are suspected to be small for gestational age [as mentioned by Naveiro-Fuentes et al. (16)], can explain the higher rates of preterm deliveries in the present study. In this study SGA neonates with iSUA had a higher rate of induction of labor compared to SGA neonates without iSUA, thought this was not found to be significant. Tul et al. suggest that SGA fetuses are delivered earlier due to maternal conditions such as the increased incidence of hypertensive disorders (18), which may cause a hostile environment for the fetus.

We found that $36.4 \%$ of SGA neonates with iSUA had been delivered via cesarean section compared with $20.3 \%$ of SGA neonates without iSUA. Raio et al. demonstrated a reduction of Wharton's jelly in cases of SUA (19). This reduction may cause the umbilical cord to be more vulnerable to compression, leading it to be more susceptible to interruptions in blood flow during contractions compromising fetal oxygenation, thus possibly explaining the higher rate of cesarean deliveries as well as the higher rates of perinatal mortality.

Furthermore, Gutvirtz et al. demonstrated a perinatal mortality rate of $2.5 \%$ among neonates born with iSUA (12), lower than was demonstrated in our study group. Another possible explanation for the higher rates of perinatal mortality in our study, relates to structural deviations of the cord which may elevate the risk of umbilical cord accidents (12). Placental pathology can be a cause for both fetal growth restriction and perinatal mortality $(20,21)$, thus may account at least in part for the increased rate of perinatal mortality. Francis et al explain a higher rate of perinatal mortality in term and post term SGA neonates due to placental "aging" (22).

While an increased risk of perinatal mortality in SGA neonates has been demonstrated in many studies $(20,22-24)$, this study is the first to look at the combination of both SGA and iSUA. These results ascertain the importance of studying these comorbidities proving these neonates to have an increased risk of developing adverse outcomes compared with neonates complicated with iSUA alone or SGA alone.

Battarbee et al. hypothesized that the cause for the higher rate of neonates with SUA being born SGA is due to the fact that they are at greater risk of abnormal placental development and perfusion (17). Bugatto et al. suggest that it is a consequence of disorders in maternal-placental circulation rather than placental insufficiency (25).

Recent studies in the field of epigenetics discuss the association between maternal environmental exposures and offspring outcomes, specifically offspring growth. Nagarajan et al. researched the effect of maternal stress on neonatal birth weight (26). They demonstrated that HSD11b2 promoter methylation, a key component in the cortisol binding pathway, was significantly higher in neonates clinically diagnosed with intrauterine growth restriction (IUGR). Kitsiou-Tzeli et al. speak of maternal nutrition, exposure to toxic substances and changes in the in-utero expression of maternally imprinted genes and their effect on neonatal and infant growth (27). One study reported on mutations in oncogenes such as PLAG1, a key factor in the IGF2 pathway, resulting in IUGR fetuses (28). Due to this, we hypothesize that such epigenetic mutations can also be expressed as an SUA. Further research must be done in this field focusing on SGA and SUA since this may contribute to our understanding of the perinatal outcomes discussed above.

The main strength of our population-based study is the large sample size allowing us to draw conclusions regarding delivering SGA neonates with and without iSUA. Additionally, this large sample size allowed us to study a specific smaller population (namely SGA neonates complicated by iSUA) in our population and its association with several clinically significant outcomes. Nevertheless, our study has a number of limitations mainly due to its retrospective design, including the potential of missing data. It must be noted that the data was reported by an obstetrician directly after delivery and was routinely reviewed by skilled medical secretaries prior to entering it into the database. Coding was done after assessing the medical prenatal care records together with the routine hospital documents. Hence this makes this potential source of selection bias less plausible. 
In conclusion, giving birth to an SGA neonate with iSUA is associated with adverse pregnancy outcomes. It is imperative to perform Intensive fetal monitoring in the presence of iSUA during delivery of a fetus suspected to be born SGA in order to decrease perinatal morbidity and mortality. Researching this particular group of neonates can be of great significance in aiding the medical team in their decision making during pregnancy and delivery of these fetuses and may possibly help prevent severe deleterious outcomes.

\section{REFERENCES}

1. Prucka S, Clemens M, Craven C, McPherson E. Single umbilical artery: what does it mean for the fetus? A case-control analysis of pathologically ascertained cases. Genet Med. (2004) 6:54-7. doi: 10.1097/01.GIM.0000105743.91723.B0

2. Csécsei K, Kovács T, Hinchliffe SA, Papp Z. Incidence and associations of single umbilical artery in prenatally diagnosed malformed, midtrimester fetuses: a review of 62 cases. Am J Med Genet. (1992) 43:524-30. doi: 10.1002/ajmg.1320430305

3. Burshtein S, Levy A, Holcberg G, Zlotnik A, Sheiner E. Is single umbilical artery an independent risk factor for perinatal mortality? Arch Gynecol Obstet. (2011) 283:191-4. doi: 10.1007/s00404-009-1326-3

4. Predanic M, Perni SC, Friedman A, Chervenak FA, Chasen ST. Fetal growth assessment and neonatal birth weight in fetuses with an isolated single umbilical artery. Obstet Gynecol. (2005) 105(5 Pt1):1093-7. doi: 10.1097/01.AOG.0000158108.51397.f5

5. Tülek F, Kahraman A, Taşkçn S, Ozkavukçu E, Söylemez F. Determination of risk factors and perinatal outcomes of singleton pregnancies complicated by isolated single umbilical artery in Turkish population. J Turk Ger Gynecol Assoc. (2015) 16:21-4. doi: 10.5152/jtgga.2015.15115

6. Baron J, Weintraub AY, Sciaky Y, Mastrolia SA, Speigel E, Hershkovitz R. Umbilical artery blood flows among pregnancies with single umbilical artery: a prospective case-control study. J Matern Fetal Neonatal Med. (2015) 28:1803-5. doi: 10.3109/14767058.2014.968845

7. Lilja M. Infants with single umbilical artery studied in a national registry. 3: a case control study of risk factors. Paediatr Perinat Epidemiol. (1994) 8:325-33.

8. Rembouskos G, Cicero S, Longo D, Sacchini C, Nicolaides KH. Single umbilical artery at 11-14 weeks' gestation: relation to chromosomal defects. Ultrasound Obstet Gynecol. (2003) 22:567-70. doi: 10.1002/uog.901

9. Lubinsky M. Embryonic hypocellularity, blastogenetic malformations, and fetal growth restriction. Am J Med Genet A. (2017) 173:151-156. doi: 10.1002/ajmg.a.37985

10. Murphy-Kaulbeck L, Dodds L, Joseph KS, Van den Hof M. Single umbilical artery risk factors and pregnancy outcomes. Obstet Gynecol. (2010) 116:84350. doi: 10.1097/AOG.0b013e3181f0bc08

11. Ashwal E, Melamed N, Hiersch L, Edel S, Bardin R, Wiznitzer A, et al. The impact of isolated single umbilical artery on labor and delivery outcome. Prenat Diagn. (2014) 34:581-5. doi: 10.1002/pd.4352

12. Gutvirtz G, Walfisch A, Beharier O, Sheiner E. Isolated single umbilical artery is an independent risk factor for perinatal mortality and adverse outcomes in term neonates. Arch Gynecol Obstet. (2016) 294:931-935. doi: 10.1007/s00404-016-4088-8

13. Zeve D, Regelmann MO, Holzman IR, Rapaport R. Small at birth, but how small? the definition of SGA revisited. Horm Res Paediatr. (2016) 86:357-360. doi: $10.1159 / 000449275$

14. Nobile S, Marchionni P, Carnielli VP. Neonatal outcome of small for gestational age preterm infants. Eur J Pediatr. (2017) 176:1083-8. doi: 10.1007/s00431-017-2957-1

15. Friebe-Hoffmann U, Hiltmann A, Friedl TWP, Lato K, Hammer R, Janni W, et al. Prenatally diagnosed single umbilical artery (SUA) - retrospective analysis of 1169 Fetuses. Ultraschall Med. (2018) doi: 10.1055/s-0043-123463. [Epub ahead of print].

16. Naveiro-Fuentes M, Carrillo-Badillo MP, Malde-Conde J, Gallo-Vallejo JL, Puertas-Prieto A. Perinatal outcomes in singleton pregnancies with a

\section{DATA AVAILABILITY}

The datasets for this manuscript are not publicly available because hospital policy. Requests to access the datasets should be directed to adiyehud@bgu.ac.il.

\section{AUTHOR CONTRIBUTIONS}

$\mathrm{MB}, \mathrm{AW}, \mathrm{YB}, \mathrm{RR}$, and GP: conception, planning, carrying out, analyzing, and writing up the work.

single umbilical artery. J Matern Fetal Neonatal Med. (2016) 29:1562-5. doi: 10.3109/14767058.2015.1053864

17. Battarbee AN, Palatnik A, Ernst LM, Grobman WA. Association of isolated single umbilical artery with small for gestational age and preterm birth. Obstet Gynecol. (2015) 126:760-4. doi: 10.1097/AOG.0000000000001037

18. Tul N, Lasic M, Bricelj K, Bregar AT, Verdenik I, Lucovnik M, et al. Outcome of small for gestational age preterm singletons: a population-based cohort study. J Perinat Med. (2016) 44:941-4. doi: 10.1515/jpm-2015-0321

19. Raio L, Ghezzi F, Di Naro E, Franchi M, Brühwiler H, Lüscher KP. Prenatal assessment of Wharton's jelly in umbilical cords with single artery. Ultrasound Obstet Gynecol. (1999) 14:42-6. doi: 10.1046/j.1469-0705.1999.14010042.x

20. Flenady V, Koopmans L, Middleton P, Frøen JF, Smith GC, Gibbons K, et al. Major risk factors for stillbirth in high-income countries: a systematic review and meta-analysis. Lancet. (2011) 377:1331-40. doi: 10.1016/S01406736(10)62233-7

21. Cnattingius S, Kramer MS, Norman M, Ludvigsson JF, Fang F, Lu D. Keep it in the family: comparing perinatal risks in small-for-gestational-age infants based on population vs within-sibling designs. Int J Epidemiol. (2018). doi: 10.1093/ije/dyy196. [Epub ahead of print].

22. Francis JH, Permezel M, Davey MA. Perinatal mortality by birthweight centile. Aust N Z J Obstet Gynaecol. (2014) 54:354-9. doi: 10.1111/ajo.12205

23. Walker AR, Waites BT, Caughey AB. Twins versus singleton pregnancies: outcomes in small for gestational age late preterm deliveries. J Matern Fetal Neonatal Med. (2018) 19:1-6. doi: 10.1080/14767058.2018.1508438

24. Hinkle SN, Sjaarda LA, Albert PS, Mendola P, Grantz KL. Comparison of methods for identifying small-for-gestational-age infants at risk of perinatal mortality among obese mothers: a hospital-based cohort study. BJOG. (2016) 123:1983-8. doi: 10.1111/1471-0528.13896

25. Bugatto F, Quintero-Prado R, Melero-Jiménez V, Fajardo-Expósito MA, Hervías-Vivancos B, Bartha JL. Ultrasound predictors of birth weight in euploid fetuses with isolated single umbilical artery. Ultrasound Obstet Gynecol. (2010) 36:724-7. doi: 10.1002/uog.7708

26. Nagarajan S, Seddighzadeh B, Baccarelli A, Wise LA, Williams M, Shields AE. Adverse maternal exposures, methylation of glucocorticoid-related genes and perinatal outcomes: a systematic review. Epigenomics. (2016) 8:925-44. doi: $10.2217 /$ epi.16.9

27. Kitsiou-Tzeli S, Tzetis M. Maternal epigenetics and fetal and neonatal growth. Curr Opin Endocrinol Diabetes Obes. (2017) 24:43-46. doi: 10.1097/MED.0000000000000305

28. Abi Habib W, Brioude F, Edouard T, Bennett JT, Lienhardt-Roussie A, Tixier F, et al. Genetic disruption of the oncogenic HMGA2-PLAG1IGF2 pathway causes fetal growth restriction. Genet Med. (2018) 20:250-8. doi: 10.1038/gim.2017.105

Conflict of Interest Statement: The authors declare that the research was conducted in the absence of any commercial or financial relationships that could be construed as a potential conflict of interest.

Copyright (๑) 2019 Blum, Weintraub, Baumfeld, Rotem and Pariente. This is an open-access article distributed under the terms of the Creative Commons Attribution License (CC BY). The use, distribution or reproduction in other forums is permitted, provided the original author(s) and the copyright owner(s) are credited and that the original publication in this journal is cited, in accordance with accepted academic practice. No use, distribution or reproduction is permitted which does not comply with these terms. 